\title{
One-pot and efficient protocol for synthesis of quinoxaline derivatives
}

\author{
Mohammad Reza Islami* and Zahra Hassani \\ Department of Chemistry, Shahid Bahonar University, Kerman, Iran \\ P.O. Box 76169-133 \\ E-mail:mrislami@mail.uk.ac.ir
}

\begin{abstract}
Efficient syntheses of quinoxalines from the reaction of $\alpha$-hydroxy ketones and $o$ phenylenediamine in acetic acid via two methods, a thermal procedure and by microwave irradiation, are described.
\end{abstract}

Keywords: Quinoxaline, $\alpha$-hydroxy ketones, $o$-phenylenediamine, heterocyclic compounds

\section{Introduction}

The synthesis and chemistry of quinoxalines have attracted considerable attention in the past ten years. ${ }^{1,2}$ Some of them exhibit biological activities including anti-viral, ${ }^{3 \mathrm{a}}$ anti-bacterial, ${ }^{3 \mathrm{~b}}$ anti-inflammatory, ${ }^{3 \mathrm{c}}$ anti-protozoal, ${ }^{3 \mathrm{~d}}$ anti-cancer ${ }^{3 \mathrm{e}}$ (colon cancer therapies), ${ }^{3 \mathrm{f}}$ antidepressant, $^{3 \mathrm{~g}}$ anti-HIV, ${ }^{3 \mathrm{~d}}$ and as kinase inhibitors. ${ }^{3 \mathrm{~h}, 3 \mathrm{i}}$ They are also used in the agricultural field as fungicides, herbicides, and insecticides. ${ }^{4}$ Also, quinoxaline moieties are present in the structure of various antibiotics such as echinomycin, levomycin and actinoleutin, which are known to inhibit the growth of gram positive bacteria and they are active against various transplantable tumors. ${ }^{5}$ In addition, quinoxaline derivatives have also found applications in dyes, ${ }^{6}$ efficient electron luminescent materials, ${ }^{4,7 a}$ organic semiconductors, ${ }^{7 b}$ chemically controllable switches, ${ }^{7 \mathrm{c}}$ building blocks for the synthesis of anion receptors, ${ }^{7 \mathrm{~d}}$ cavitands, ${ }^{7 \mathrm{e}}$ and dehydoannulenes. ${ }^{7 f}$ They also serve as useful rigid subunits in macrocyclic receptors in molecular recognition. ${ }^{6 a}$

Numerous methods are available for the synthesis of quinoxaline derivatives which involve condensation of 1,2-diamines with $\alpha$-diketones, ${ }^{8} 1,4$-addition of 1 ,2-diamines to diazenylbutenes, ${ }^{9}$ cyclization-oxidation of phenacyl bromides ${ }^{10}$ and oxidative coupling of epoxides with ene-1,2-diamines. ${ }^{11}$ 2,3-Disubstituted quinoxalines have also been prepared via the Suzuki-Miyaura coupling reaction, ${ }^{12}$ condensation of $o$-phenylenediamines with 1,2dicarbonyl compounds in $\mathrm{MeOH} / \mathrm{AcOH}$ under microwave irradiation, ${ }^{13}$ and iodine catalyzed cyclocondensation of 1,2-dicarbonyl compounds with substituted $o$-phenylenediamines in $\mathrm{DMSO}^{14}$ or $\mathrm{CH}_{3} \mathrm{CN}^{15}$ Also, $\alpha$-hydroxy ketones react with $o$-phenylenediamines in the presence of transition metals such as $\mathrm{Mn}, \mathrm{Pd}, \mathrm{Ru}$ and $\mathrm{Cu}, \mathrm{Pb}$ to give quinoxalines. ${ }^{11,16}$ The 
most common method is the condensation of an aryl 1,2-diamine with a 1,2-dicarbonyl compound by heating in a solvent for $2-12 \mathrm{~h}$. The yields of products are between $34-85 \%{ }^{8 \mathrm{a}}$ Improved methods have been reported for the synthesis of quinoxaline derivatives including a Bi-catalyzed oxidative coupling method, ${ }^{11}$ a microwave procedure, ${ }^{13}$ and the use of $\mathrm{RuCl}_{2}$ $\left(\mathrm{PPh}_{3}\right)_{3}$-TEMPO, ${ }^{16 \mathrm{a}} \mathrm{MnO}_{2},{ }^{16 \mathrm{~b}} \mathrm{POCl}_{3},{ }^{16 \mathrm{f}}$ zeolites, ${ }^{14}$ iodine, ${ }^{16 \mathrm{~g}}$ cerium ammonium nitrate, ${ }^{16 \mathrm{~h}}$ $\mathrm{CuSO}_{4} .5 \mathrm{H}_{2} \mathrm{O},{ }^{16 \mathrm{i}} \quad$ Montmorillonite $\mathrm{K}-10,{ }^{16 \mathrm{j}} \quad \mathrm{H}_{6} \mathrm{P}_{2} \mathrm{~W}_{18} \mathrm{O}_{62} .24 \mathrm{H}_{2} \mathrm{O}$; Wells-Dawson ${ }^{16 \mathrm{k}}$ and $\mathrm{SA} / \mathrm{MeOH}^{161}$ as a catalyst. However, many of these processes suffer from one or more limitations such as drastic reaction conditions, low product yields, tedious work-up procedures, the use of toxic metal salts as catalysts, and relatively expensive reagents. Moreover, these reactions are often carried out in polar solvents such as DMSO leading to tedious work-up procedures. Recently, Juncai and co-workers ${ }^{17}$ used o-phenylenediamines and $\alpha$-hydroxy ketones as reactants in the synthesis of quinoxaline derivatives. In all cases, they were obtained aromatic quinoxalines. We were interested to examine the synthesis of quinoxalines by heating in acetic acid and under the influence of microwave irradiation and we report here that this led to a change in the results and gave rise to novel quinoxalines.

\section{Result and Discussion}

Recently we have developed new and powerful methods for the synthesis of pyrroloisoindoles, ${ }^{18}$ pyrazoloisoindoles ${ }^{19}$ and pyrrolizines. ${ }^{20}$ Now we have investigated the synthesis quinoxalines using of $o$-phenylenediamine and $\alpha$-hydroxy ketones as reactants in acetic acid via two methods: microwave irradiation and simple heating. As outlined in Scheme 1 and Table 1, the reaction of $o$-phenylenediamine and $\alpha$-hydroxy ketones 2a-e in refluxing acetic acid gave only compounds $4 a-e$ and the same reaction with $\alpha$-hydroxy ketones $\mathbf{2 f}, \mathbf{g}$ gave only compounds $\mathbf{3 f}, \mathbf{g}$.

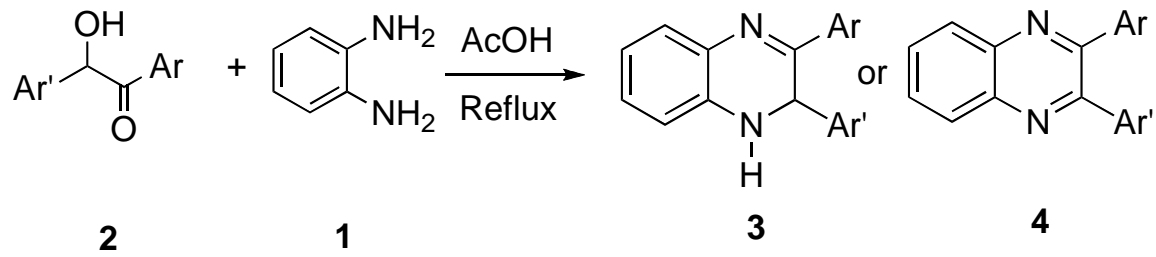

\section{Scheme 1}

Some representative results are summarized in Table 1, and the following procedure is typical: a mixture of $o$-phenylenediamine $\mathbf{1}$ and benzoin 2a in acetic acid was heated to reflux for $2 \mathrm{~h}$. After cooling to room temperature, the reaction mixture was poured in to cold water, extracted with dichloromethane, the extract dried with calcium chloride, and the solvent evaporated under reduced pressure to afford the crude product. 2,3-Diphenylquinoxaline 4a was obtained after recrystalisation in ethanol as white needles. Other $\alpha$-hydroxy ketones $\mathbf{2 b}$-e were reacted analogously to afford compounds $\mathbf{4 b - e}$ in $83-91 \%$ yields. Only compounds $\mathbf{2 f}$,g were converted products $\mathbf{3 f}$,g. 
Table 1. Preparation of quinoxalines in acetic acid via two different methods

\begin{tabular}{|c|c|c|c|c|c|c|}
\hline \multirow{3}{*}{ Compound } & \multirow{3}{*}{$\mathrm{Ar}$} & \multirow{3}{*}{$\mathrm{Ar}^{\prime}$} & \multicolumn{2}{|c|}{ Thermal method } & \multicolumn{2}{|c|}{ Microwave method $^{b}$} \\
\hline & & & \multicolumn{2}{|c|}{ Yields $(\%)$} & \multicolumn{2}{|c|}{ Yields (\%) } \\
\hline & & & 3 & 4 & 3 & 4 \\
\hline $2 a$ & $\mathrm{Ph}$ & $\mathrm{Ph}$ & - & 96 & - & 98 \\
\hline $2 \mathbf{b}$ & $p-\mathrm{CH}_{3} \mathrm{OC}_{6} \mathrm{H}_{4}$ & $p-\mathrm{CH}_{3} \mathrm{OC}_{6} \mathrm{H}_{4}$ & - & 88 & 97 & - \\
\hline 2c & $p-\mathrm{CH}_{3} \mathrm{C}_{6} \mathrm{H}_{4}$ & $p-\mathrm{CH}_{3} \mathrm{C}_{6} \mathrm{H}_{4}$ & - & 91 & 96 & - \\
\hline 2d & $p-\mathrm{Me}_{2} \mathrm{NC}_{6} \mathrm{H}_{4}$ & $p-\mathrm{ClC}_{6} \mathrm{H}_{4}$ & - & 83 & - & 97 \\
\hline $2 e$ & $p-\mathrm{CH}_{3} \mathrm{OC}_{6} \mathrm{H}_{4}$ & $\mathrm{Ph}$ & - & 90 & - & 92 \\
\hline $2 f$ & $p-\mathrm{Me}_{2} \mathrm{NC}_{6} \mathrm{H}_{4}$ & $2,4-\mathrm{Cl}_{2} \mathrm{C}_{6} \mathrm{H}_{3}$ & 85 & - & 92 & - \\
\hline $2 g$ & $p-\mathrm{Me}_{2} \mathrm{NC}_{6} \mathrm{H}_{4}$ & $\mathrm{Ph}$ & 92 & - & 96 & - \\
\hline
\end{tabular}

${ }^{a}$ Reaction conditions: refluxing acetic acid for $2 \mathrm{~h}$.

${ }^{\mathrm{b}}$ Reaction conditions: microwave irradiation $(\mathrm{P}=450 \mathrm{~W})$ in acetic acid for $3 \mathrm{~min}$.

Under microwave irradiation, compounds $\mathbf{2 a}, \mathbf{2 d}$, and $\mathbf{2 e}$ were converted into the desired products $4 \mathbf{a}, \mathbf{4 d}$ and $\mathbf{4 e}$. When $\alpha$-hydroxy ketones $\mathbf{2 b}, \mathbf{2 c}, \mathbf{2 f}$ and $\mathbf{2 g}$ were used as substrates, reactions did not proceed to completion and products $\mathbf{3 b}, \mathbf{3 c}, \mathbf{3} \mathbf{f}$ and $\mathbf{3 g}$ were obtained. The reason of this diffference is not clear to us, but it is important to note that we used acetic acid as a solvent in both methods. It has been shown ${ }^{21}$ that when acetic acid is used as a solvent in the microwave conditions, it does not increase the temperature of the reaction mixture. This is true for the other solvents such as water, ethanol and formic acid because the loss factors ( $\tan$ $\delta$ ) of these solvents are high but in the case of acetic acid it is medium (0.174).

We speculate then that under microwave conditions, the energy required for aromatization is not available for all of the compounds to aromatize. To support this idea, when compounds $\mathbf{3 b}, \mathbf{c}$ which were obtained in microwave conditions, were refluxed in acetic acid they were easily converted into $\mathbf{4 b , c}$ after $60 \mathrm{~min}$. A reasonable mechanism is suggested in Scheme 2.

The structures of products were assigned based on the IR, ${ }^{1} \mathrm{H} N M R$ and ${ }^{13} \mathrm{C}$ NMR spectra along with elemental $\mathrm{CHN}$-analyses data for unknown compounds. In the IR spectra of compounds 3b, 3c, 3f and $\mathbf{3 g}$, the NH absorption band appeared at $3329 \mathrm{~cm}^{-1}$, whereas in the IR spectra of compound 4a-e this absorption band was not observed. The ${ }^{1} \mathrm{H}$ NMR spectra of compounds 3b, 3c, 3f and 3g showed a singlet peak at $\delta 4.41-4.71 \mathrm{ppm}$ for methine proton along with a broad signal bond at $\delta$ 5.57- $6.09 \mathrm{ppm}$ for the $\mathrm{NH}$ group. The ${ }^{13} \mathrm{C} \mathrm{NMR}$ spectra were in agreement with the proposed structures (see Experimental Section). 


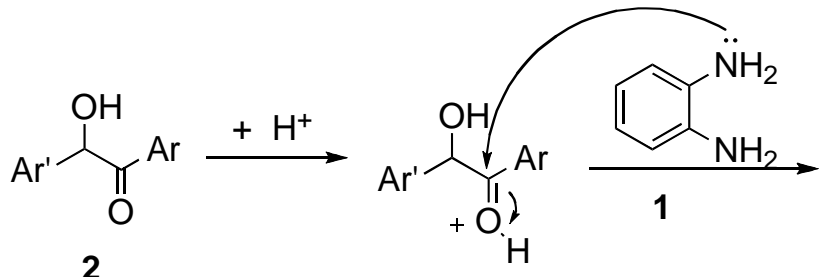

2
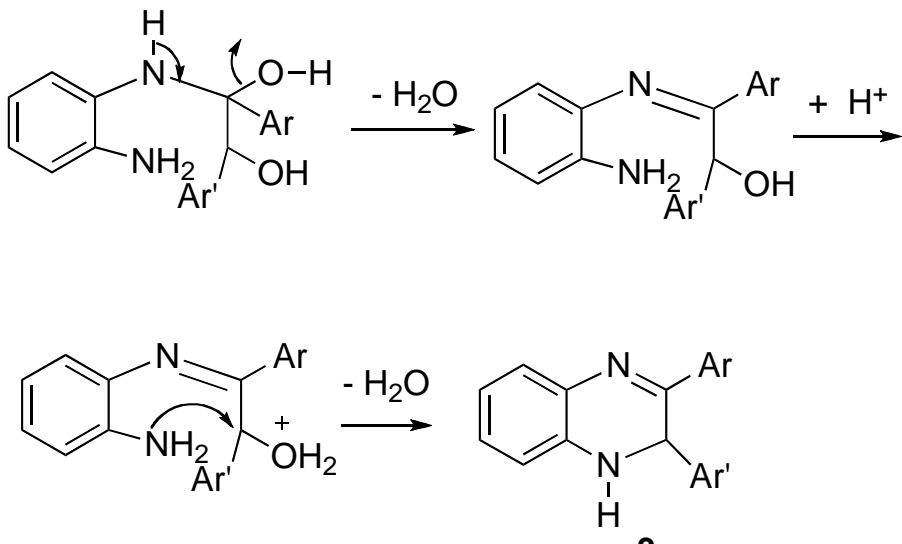

3<smiles>Brc1nc2ccccc2nc1Br</smiles>

4

\section{Scheme 2}

\section{Conclusions}

In conclusion, we have developed two straightforward cost-effective methods for the synthesis of quinoxalines in good to excellent yields. The reaction time in microwave irradiation was reduced in comparison to thermal procedure however in some cases, aromatization of the immediate dihydroquinoxaline products did not occur.

\section{Experimental Section}

General Procedures. Melting points were obtained on a Gallenkamp melting point apparatus and are uncorrected. IR spectra were recorded on a Mattson 1000 FT-IR spectrometer. The ${ }^{1} \mathrm{H}$, and ${ }^{13} \mathrm{C}$ NMR spectra were recorded on a BRUKER DRX-500 AVANCE $\left({ }^{1} \mathrm{H}\right.$ at 500 $\mathrm{MHz}$, and ${ }^{13} \mathrm{C}$ at $125.77 \mathrm{MHz}$ ). Elemental analysis for $\mathrm{C}, \mathrm{H}$ and $\mathrm{N}$ were determined by using a Heracus CHN-O-Rapid analyzer by the chemistry department laboratory located at Tarbiat Moallem University. Chemicals were employed as purchased from Merck, Fluka and Sigma. Solvents such as ethanol, glacial acetic acid, dichloromethane and diethyl ether used without purification. o-Phenylenediamine was recrystallised from hot ethanol. Benzaldehyde, $p$ methylbenzaldehyde, $\quad p$-methoxybenzaldehyde, $\quad p$-N,N-dimethylbenzaldehyde, $\quad p$ - 
chlorobenzaldehyde and 2,4-dichlorobenzaldehyde used in the preparation of benzoins ${ }^{22}$ were commercially available and were used after purification.

\section{General procedures for the synthesis of 2,3-diphenylquinoxaline(4a). Thermal method} A mixture of benzoin $(0.42 \mathrm{~g}, 2 \mathrm{mmol})$, o-phenylenediamine $(0.22 \mathrm{~g}, 2 \mathrm{mmol})$ and $10 \mathrm{~mL}$ of glacial acetic acid was heated to reflux. After 5 minutes a brown solution was observed; the progress of the reaction was monitored by TLC, and reflux was continued for $2 \mathrm{~h}$. After cooling to room temperature, the reaction mixture was poured into cold water, extracted with dichloromethane $(3 \times 10 \mathrm{~mL})$, dried with anhydrous calcium chloride, and then the solvent was evaporated under reduced pressure to afford the crude product. 2,3-Diphenylquinoxaline 4a was obtained as white needles after recrystalisation from ethanol. (0.55 g, m.p. 127-128 ${ }^{\circ} \mathrm{C}$ [reported m.p. $\left.128-129^{\circ} \mathrm{C}\right]^{5 b, 16 e, i, k}$, yield 98\%), IR (KBr) $\left(v \max , \mathrm{cm}^{-1}\right): 3055,1542,1495$, 1448, 1355. ${ }^{1} \mathrm{H} \mathrm{NMR}\left(\mathrm{CDCl}_{3}, 500 \mathrm{MHz}\right) \delta 7.33-7.37(\mathrm{~m}, 3 \mathrm{H}), 7.56(\mathrm{dd}, 2 \mathrm{H}, J=7.8 \mathrm{~Hz}, 1.2$ $\mathrm{Hz}), 7.76(\mathrm{dd}, 1 \mathrm{H}, J=6.3 \mathrm{~Hz}, 3.4 \mathrm{~Hz}), 8.2(\mathrm{dd}, 1 \mathrm{H}, J=6.3 \mathrm{~Hz}, 3.4 \mathrm{~Hz}) \mathrm{ppm} .{ }^{13} \mathrm{C} \mathrm{NMR}(125$ $\left.\mathrm{MHz}, \mathrm{CDCl}_{3}\right) \delta 128.27(\mathrm{CH}), 128.81(\mathrm{CH}), 129.25(\mathrm{CH}), 129.91(\mathrm{CH}), 129.92(\mathrm{CH}), 139.18$ (C), $141.28(\mathrm{C}), 153.46$ (C) ppm.

\section{Microwave irradiation method}

A mixture of benzoin $(0.42 \mathrm{~g}, 2 \mathrm{mmol})$, o-phenylenediamine $(0.22 \mathrm{~g}, 2 \mathrm{mmol})$, and $2 \mathrm{~mL}$ glacial acetic acid was irradiated in a microwave oven at a power output of $450 \mathrm{~W}$. After 10 seconds, a brown solution was observed; the progress of the reaction was monitored by TLC. After completion of the reaction (upon three minutes), the reaction mixture was poured into cold water, extracted with dichloromethane $(3 \times 10 \mathrm{~mL})$, the extract dried with calcium chloride, and then the solvent was evaporated under reduced pressure to afford the crude product. 2,3-Diphenylquinoxaline 4a was obtained as white needles after recrystalisation from ethanol. (0.54 g, m.p. $127-128^{\circ} \mathrm{C}$ [reported m.p. $\left.128-129^{\circ} \mathrm{C}\right]^{5 b, 16 e, i, k}$, yield $\left.96 \%\right)$.

2,3-Di(4-methoxyphenyl)quinoxaline (4b). (0.60 g, m.p. $151-152{ }^{\circ} \mathrm{C}$ [reported m.p. 151$\left.152.5^{\circ} \mathrm{C}\right],{ }^{5 \mathrm{~b}, 16 \mathrm{e}, \mathrm{i}, \mathrm{k}, 17}$ yield 88\%), IR (KBr) $\left(v \max , \mathrm{cm}^{-1}\right): 3056,2957,1617,1517,1448,1355$. ${ }^{1} \mathrm{H} \mathrm{NMR}\left(500 \mathrm{MHz}, \mathrm{CDCl}_{3}\right) \delta 3.83\left(\mathrm{~s}, 3 \mathrm{H}, \mathrm{OCH}_{3}\right), 6.89(\mathrm{~d}, 2 \mathrm{H}, J=8.3 \mathrm{~Hz}), 7.52(\mathrm{~d}, 2 \mathrm{H}, J=$ $8.3 \mathrm{~Hz}), 7.72(\mathrm{dd}, 1 \mathrm{H}, J=6.1 \mathrm{~Hz}, 3.2 \mathrm{~Hz}), 8.14(\mathrm{dd}, 1 \mathrm{H}, J=6.05 \mathrm{~Hz}, 3.2 \mathrm{~Hz}) \mathrm{ppm} .{ }^{13} \mathrm{C} \mathrm{NMR}$ $\left(125 \mathrm{MHz}, \mathrm{CDCl}_{3}\right) \delta 55.30\left(\mathrm{OCH}_{3}\right), 113.79(\mathrm{CH}), 129.03(\mathrm{CH}), 129.49(\mathrm{CH}), 131.29(\mathrm{CH})$, 131.79 (C), 141.09 (C), 153.00 (C), 160.21(C) ppm.

2,3-Di-p-tolylquinoxaline (4c). (0.57 g, m.p. 149-150 ${ }^{\circ} \mathrm{C}$ [reported m.p. $145-146{ }^{\circ} \mathrm{C}$ ], ${ }^{14,16 j, 16 \mathrm{~m}}$ yield 91\%), IR (KBr) $\left(v \max , \mathrm{cm}^{-1}\right): 3031,2958,1617,1517,1448,1355 .{ }^{1} \mathrm{H}$ NMR $(500$ $\left.\mathrm{MHz}, \mathrm{CDCl}_{3}\right) \delta 2.40\left(\mathrm{~s}, 3 \mathrm{H}, \mathrm{CH}_{3}\right), 7.18(\mathrm{~d}, 2 \mathrm{H}, J=7.9 \mathrm{~Hz}), 7.48$ (d, 2H, $\left.J=7.9 \mathrm{~Hz}\right), 7.76$ $(\mathrm{dd}, 1 \mathrm{H}, J=6.3 \mathrm{~Hz}, 3.4 \mathrm{~Hz}), 8.19(\mathrm{dd}, 1 \mathrm{H}, J=6.3 \mathrm{~Hz}, 3.4 \mathrm{~Hz}) \mathrm{ppm} .{ }^{13} \mathrm{C}$ NMR $(125 \mathrm{MHz}$, $\left.\mathrm{CDCl}_{3}\right) \delta 21.36\left(\mathrm{CH}_{3}\right), 128.99(\mathrm{CH}), 129.15(\mathrm{CH}), 129.64(\mathrm{CH}), 129.78(\mathrm{CH}), 136.47(\mathrm{C})$, $138.74(\mathrm{C}), 141.20(\mathrm{C}), 153.49(\mathrm{C}) \mathrm{ppm}$.

4-(2-(4-Chlorophenyl)quinoxalin-3-yl)- $N, N$-dimethylbenzenamine (4d). (0.56 g, m.p. $168-170{ }^{\circ} \mathrm{C}$, yield $\left.83 \%\right)$, IR (KBr) $\left(v \max , \mathrm{cm}^{-1}\right): 3056,2932,1617,1542,1448,1355 .{ }^{1} \mathrm{H}$ NMR (500 MHz, $\left.\mathrm{CDCl}_{3}\right) \delta 3.01\left(\mathrm{~s}, 3 \mathrm{H}, 2 \mathrm{CH}_{3}\right), 6.67(\mathrm{dd}, 1 \mathrm{H}, J=10.0 \mathrm{~Hz}, 2.5 \mathrm{~Hz}), 7.37$ (dd, $1 \mathrm{H}, J=5.0 \mathrm{~Hz}, 2.5 \mathrm{~Hz}), 7.46(\mathrm{dd}, 1 \mathrm{H}, J=5.0 \mathrm{~Hz}, 2.5 \mathrm{~Hz}), 7.57(\mathrm{dd}, 1 \mathrm{H}, J=10.0 \mathrm{~Hz}, 2.5 \mathrm{~Hz})$, 7.71-7.75 (m, 1H), 8.11-8.14 (m, CH) ppm. ${ }^{13} \mathrm{C}$ NMR (125 MHz, $\left.\mathrm{CDCl}_{3}\right) \delta 40.00\left(\mathrm{CH}_{3}\right)$, 
$111.74(\mathrm{CH}), 125.99(\mathrm{C}), 128.55(\mathrm{CH}), 128.96(\mathrm{CH}), 129.04(\mathrm{CH}), 129.16(\mathrm{CH}), 129.86$ $(\mathrm{CH}), 131.09(\mathrm{CH}), 131.11(\mathrm{CH}), 134.77(\mathrm{C}), 138.50(\mathrm{C}), 140.62(\mathrm{C}), 141.56(\mathrm{C}), 150.86(\mathrm{C})$, 152.16 (C), 153.29 (C) ppm. Anal. Calcd. For $\mathrm{C}_{22} \mathrm{H}_{18} \mathrm{ClN}_{3}$ (359.85): C, 73.43; H, 5.04; N, 11.68\%. Found: C, 73.23; H, 5.15; N, 11.56\%.

2-(4-Methoxyphenyl)-3-phenylquinoxaline (4e). (0.56 g, m.p. $118-120{ }^{\circ} \mathrm{C}$ [reported $]^{12}$, yield 90\%), IR (KBr) $\left(v \max , \mathrm{cm}^{-1}\right): 3056,2957,1617,1517,1448,1355 .{ }^{1} \mathrm{H}$ NMR $(500$ $\left.\mathrm{MHz} \mathrm{CDCl}_{3}\right) \delta 3.84\left(\mathrm{~s}, 3 \mathrm{H}, \mathrm{OCH}_{3}\right), 6.88(\mathrm{td}, 2 \mathrm{H}, J=8.8 \mathrm{~Hz}, 1.8 \mathrm{~Hz}), 7.39(\mathrm{dd}, 3 \mathrm{H}, J=5.9$ $\mathrm{Hz}, 1.25 \mathrm{~Hz}), 7.50-7.58(\mathrm{~m}, 4 \mathrm{H}), 7.76-7.78(\mathrm{~m}, 2 \mathrm{H}), 8.18(\mathrm{dd}, 1 \mathrm{H}, J=5.6 \mathrm{~Hz}, 2.7 \mathrm{~Hz}) \mathrm{ppm}$. ${ }^{13} \mathrm{C}$ NMR $\left(125 \mathrm{MHz}, \mathrm{CDCl}_{3}\right) \delta 55.30\left(\mathrm{OCH}_{3}\right), 113.75(\mathrm{CH}), 128.33(\mathrm{CH}), 128.73(\mathrm{CH})$, $129.03(\mathrm{CH}), 129.09(\mathrm{CH}), 129.18(\mathrm{CH}), 129.58(\mathrm{CH}), 129.76(\mathrm{CH}), 129.83(\mathrm{CH}), 131.37$ (C), 139.48 (C), 141.03 (C), 141.33 (C), 153.04 (C), 153.43 (C), 160.26 (C) ppm.

1,2-Dihydro-2,3-di(4-methoxyphenyl)quinoxaline (3b). (0.67 g, m.p. 165-166 ${ }^{\circ} \mathrm{C}$, yield 97\%), IR (KBr) $\left(v \max , \mathrm{cm}^{-1}\right): 3329,3056,2957,1612,1565,1519,1448,1355 .{ }^{1} \mathrm{H}$ NMR $\left(500 \mathrm{MHz}, \mathrm{CDCl}_{3}\right) \delta 3.69\left(\mathrm{~s}, 3 \mathrm{H}, \mathrm{OCH}_{3}\right), 3.79\left(\mathrm{~s}, 3 \mathrm{H}, \mathrm{OCH}_{3}\right), 4.55(\mathrm{~s}, 1 \mathrm{H}, \mathrm{CH}), 5.57(\mathrm{~s}, 1 \mathrm{H}$, $\mathrm{NH}), 6.75(\mathrm{td}, 2 \mathrm{H}, J=8.7 \mathrm{~Hz}, 2.0 \mathrm{~Hz}), 6.82(\mathrm{td}, 1 \mathrm{H}, J=6.9 \mathrm{~Hz}, 1.2 \mathrm{~Hz}), 6.88(\mathrm{td}, 2 \mathrm{H}, J=8.9$ $\mathrm{Hz}, 1.9 \mathrm{~Hz}), 6.99$ (td, 2H, $J=6.9 \mathrm{~Hz}, 1.4 \mathrm{~Hz}), 7.17(\mathrm{td}, 2 \mathrm{H}, J=8.7 \mathrm{~Hz}, 2.0 \mathrm{~Hz}), 7.74(\mathrm{dd}, 2 \mathrm{H}$, $J=6.4 \mathrm{~Hz}, 3.4 \mathrm{~Hz}), 7.93(\mathrm{td}, 2 \mathrm{H}, J=8.9 \mathrm{~Hz}, 1.9 \mathrm{~Hz}), 8.18(\mathrm{dd}, 2 \mathrm{H}, J=6.4 \mathrm{~Hz}, 3.4 \mathrm{~Hz}) \mathrm{ppm}$. ${ }^{13} \mathrm{C}$ NMR $\left(125 \mathrm{MHz}, \mathrm{CDCl}_{3}\right) \delta 53.94(\mathrm{CH}), 55.16\left(\mathrm{OCH}_{3}\right), 55.31\left(\mathrm{OCH}_{3}\right), 113.83(\mathrm{CH})$, $118.80(\mathrm{CH}), 127.74(\mathrm{CH}), 127.92(\mathrm{CH}), 128.18(\mathrm{CH}), 128.86(\mathrm{CH}), 129.05(\mathrm{CH}), 131.35$ (CH), 133.37 (C), 135.08 (C), 141.13 (C), 157.42 (C), 159.47 (C), 160.25 (C), 161.34 (C) ppm. Anal. Calcd. For $\mathrm{C}_{22} \mathrm{H}_{20} \mathrm{~N}_{2} \mathrm{O}_{2}$ (344.42): C, 76.72; H, 5.85; N, 8.13\%. Found: C, 76.74; H, $6.04 ; \mathrm{N}, 8.20 \%$.

1,2-Dihydro-2,3-di-p-tolylquinoxaline (3c). (0.60 g m.p. $138-140{ }^{\circ} \mathrm{C}$, yield $96 \%$ ), IR (KBr) $\left(v \max , \mathrm{cm}^{-1}\right): 3329,3031,2958,1612,1519,1495,1355 .{ }^{1} \mathrm{H} \mathrm{NMR}\left(500 \mathrm{MHz}, \mathrm{CDCl}_{3}\right) \delta 2.28$ (s, 3H, $\left.\mathrm{CH}_{3}\right), 2.38\left(\mathrm{~s}, 3 \mathrm{H}, \mathrm{CH}_{3}\right), 4.47(\mathrm{~s}, 1 \mathrm{H}, \mathrm{CH}), 5.64(\mathrm{~s}, 1 \mathrm{H}, \mathrm{NH}), 6.50(\mathrm{td}, 1 \mathrm{H}, J=7.8 \mathrm{~Hz}$, $1.2 \mathrm{~Hz}), 6.83(\mathrm{td}, 1 \mathrm{H}, J=7.5 \mathrm{~Hz}, 1.2 \mathrm{~Hz}), 7.02(\mathrm{td}, 1 \mathrm{H}, J=7.6 \mathrm{~Hz}, 1.4 \mathrm{~Hz}), 7.07$ (d, 2H, $J=$ $7.9 \mathrm{~Hz}), 7.49$ (td, $1 \mathrm{H}, J=7.9 \mathrm{~Hz}, 1.2 \mathrm{~Hz}), 7.77(\mathrm{dd}, 2 \mathrm{H}, J=6.4 \mathrm{~Hz}, 3.0 \mathrm{~Hz}), 7.88(\mathrm{~d}, 2 \mathrm{H}, J=$ $8.2 \mathrm{~Hz}), 8.20(\mathrm{dd}, 2 \mathrm{H}, J=6.5 \mathrm{~Hz}, 3.0 \mathrm{~Hz}) \mathrm{ppm} .{ }^{13} \mathrm{C} \mathrm{NMR}\left(125 \mathrm{MHz}, \mathrm{CDCl}_{3}\right) \delta 21.36\left(\mathrm{CH}_{3}\right)$, $21.41\left(\mathrm{CH}_{3}\right), 54.43(\mathrm{CH}), 113.78(\mathrm{CH}), 118.8(\mathrm{CH}), 126.58(\mathrm{CH}), 127.16(\mathrm{CH}), 127.98(\mathrm{CH})$, $128.39(\mathrm{CH}), 129.01(\mathrm{CH}), 129.16(\mathrm{CH}), 135.12(\mathrm{C}), 136.48(\mathrm{C}), 137.96(\mathrm{C}), 138.78(\mathrm{C})$, 140.46 (C), 141.22 (C), 157.84 (C) ppm. Anal. Calcd. For $\mathrm{C}_{22} \mathrm{H}_{20} \mathrm{~N}_{2}$ (312.42): C, 84.85; H, $6.45 ; \mathrm{N}, 8.97 \%$. Found: C, 84.66; H, 6.58; N, 9.00\%.

4-[2-(2,4-Dichlorophenyl)-1,2-dihydroquinoxalin-3-yl]- $\boldsymbol{N}, \boldsymbol{N}$-dimethylbenzenamine (3f). (0.73 g, m.p. $178-180^{\circ} \mathrm{C}$, yield 92\%), IR (KBr) $\left(v \max , \mathrm{cm}^{-1}\right): 3329,3056,2957,1612,1589$, 1542. ${ }^{1} \mathrm{H}$ NMR (500 MHz, $\left.\mathrm{CDCl}_{3}\right) \delta 3.02\left(\mathrm{~s}, 6 \mathrm{H}, 2 \mathrm{CH}_{3}\right), 4.71(\mathrm{CH}), 6.09(\mathrm{~s}, 1 \mathrm{H}, \mathrm{NH}), 6.10$ $(\mathrm{d}, 1 \mathrm{H}, J=1.9 \mathrm{~Hz}), 6.51(\mathrm{dd}, 1 \mathrm{H}, J=7.8 \mathrm{~Hz}, 0.9 \mathrm{~Hz}), 6.68(\mathrm{~d}, 2 \mathrm{H}, J=9.0 \mathrm{~Hz}), 6.82(\mathrm{td}, 1 \mathrm{H}$, $J=7.8 \mathrm{~Hz}, 1.0 \mathrm{~Hz}), 6.98(\mathrm{td}, 1 \mathrm{H}, J=7.8 \mathrm{~Hz}, 1.2 \mathrm{~Hz}), 7.02(\mathrm{~d}, 1 \mathrm{H}, J=2.7 \mathrm{~Hz}), 6.98-7.02(\mathrm{~m}$, $3 \mathrm{H}), 7.45(2 \mathrm{H}, \mathrm{d}, J=7.6 \mathrm{~Hz}), 7.8(2 \mathrm{H}, \mathrm{d}, J=9.0 \mathrm{~Hz}) \mathrm{ppm} .{ }^{13} \mathrm{C}$ NMR $\left(125 \mathrm{MHz}, \mathrm{CDCl}_{3}\right) \delta$ $40.06\left(2 \mathrm{CH}_{3}\right), 50.06(\mathrm{CH}), 111.50(\mathrm{CH}), 113.89(\mathrm{CH}), 119.27(\mathrm{CH}), 123.86(\mathrm{C}), 127.47$ $(\mathrm{CH}), 127.60(\mathrm{CH}), 127.92(\mathrm{CH}), 128.36(\mathrm{CH}), 129.86(\mathrm{CH}), 130.33(\mathrm{CH}), 132.33(\mathrm{C})$, 133.74 (C), 134.14 (C), 134.48 (C), 135.25 (C), 151.93 (C), 156.16 (C) ppm. Anal. Calcd. for $\mathrm{C}_{22} \mathrm{H}_{19} \mathrm{Cl}_{2} \mathrm{~N}_{3}$ (396.32): C, 66.67; H, 4.83; N, 10.60\%. Found: C, 66.54; H, 4.78; N, 10.53\%. 
4-(1,2-Dihydro-2-phenylquinoxalin-3-yl)- $N, N$-dimethylbenzenamine (3g). (0.63 g, m.p. 190-192 ${ }^{\circ} \mathrm{C}$, yields 96\%), IR (KBr) $\left(v \max , \mathrm{cm}^{-1}\right): 3329,3056,2957,1612,1565,1519,1495$. ${ }^{1} \mathrm{H}$ NMR $\left(500 \mathrm{MHz}, \mathrm{CDCl}_{3}\right) \delta 3.01\left(\mathrm{~s}, 6 \mathrm{H}, 2 \mathrm{CH}_{3}\right), 4.41(\mathrm{CH}), 5.68(\mathrm{~s}, 1 \mathrm{H}, \mathrm{NH}), 6.50(\mathrm{~d}, 1 \mathrm{H}$, $J=7.7 \mathrm{~Hz}), 6.68(\mathrm{~d}, 2 \mathrm{H}, J=9.6 \mathrm{~Hz}), 6.81(\mathrm{td}, 1 \mathrm{H}, J=7.6 \mathrm{~Hz}, 1.2 \mathrm{~Hz}), 6.98$ (td, $1 \mathrm{H}, J=7.6$ $\mathrm{Hz}, 1.2 \mathrm{~Hz}), 7.21-7.30(\mathrm{~m}, 5 \mathrm{H}), 7.44(\mathrm{~d}, 1 \mathrm{H}, J=7.7 \mathrm{~Hz}), 7.90(\mathrm{~d}, 2 \mathrm{H}, J=9.6 \mathrm{~Hz}) \mathrm{ppm} .{ }^{13} \mathrm{C}$ NMR (125 MHz, $\left.\mathrm{CDCl}_{3}\right) \delta 40.08\left(2 \mathrm{CH}_{3}\right), 54.42(\mathrm{CH}), 111.44(\mathrm{CH}), 113.68(\mathrm{CH}), 118.93$ $(\mathrm{CH}), 124.75(\mathrm{C}), 126.66(\mathrm{CH}), 127.37(\mathrm{CH}), 127.98(\mathrm{CH}), 128.65(\mathrm{CH}), 129.08(\mathrm{CH})$, $133.86(\mathrm{CH}), 134.78(\mathrm{C}), 141.28(\mathrm{C}), 145.10(\mathrm{C}), 151.71(\mathrm{C}), 157.64(\mathrm{C}) \mathrm{ppm}$. Anal. Calcd. for $\mathrm{C}_{22} \mathrm{H}_{21} \mathrm{~N}_{3}$ (327.43): C, 80.70; H, 6.46; N, 12.83\%. Found: C, 80.58; H, 6.59; N, 12.58\%.

\section{Acknowledgements}

We thank the Shahid Bahonar University for financial support as a Grant-in-Aid for Scientific Research.

\section{References}

1. Katritzky, A. R.; Rees, C.W. Comprehensive Heterocyclic Chemistry, Pergamon: Oxford, Part 2B, 1984; Vol. 3, p 157.

2. Sherman, D.; Kawakami, J.; He, H. Y.; Dhun, F.; Rios, R.; Liu, H.; Pan, W.; Xu, Y. J.; Hong, S. P.; Arbour, M.; Labelle M.; Duncton, M. A. J. Tetrahedron Lett. 2007, 48, 8943.

3. (a) Loriga, M.; Piras, S.; Sanna, P.; Paglietti, G. Farmaco. 1997, 52, 157. (b) Seitz, L. E.; Suling, W. J.; Reynolds, R. C. J. Med. Chem. 2002, 45, 5604. (c) YB, K.; YH, K.; JY, P.; SK, K. Bioorg. Med. Chem. Lett. 2004, 14, 541. (d) Hui, X.; Desrivot, J.; Bories, C.; Loiseau, P. M.; Franck, X.; Hocquemiller, R.; Figadere, B. Bioorg. Med. Chem. Lett. 2006, 16, 815. (e) Lindsley, C. W.; Zhao, Z.; Leister, W. H.; Robinson, R. G.; Barnett, S. F.; Defeo-Jones, D.; Jones, R. E.; Hartman, G. D.; Huff, J. R.; Huber, H. E.; Duggan, M. E. Bioorg. Med. Chem. Lett. 2005, 15, 761. (f) Labarbera, D. V.; Skibo, E. B. Bioorg. Med. Chem. 2005, 13, 387. (g) Sarges, R.; Howard, H. R.; Browne, R. G.; Lebel, L. A.; Seymour, P. A.; Koe, B. K. J. Med. Chem. 1990, 33, 2240. (h) Srinivas, C.; Kumar, C. N. S. S. P.; Rao, V. J.; Palaniappan, S. J. Mol. Catal. A: Chem. 2007, 265, 227. (i) Ghomsi, N. T.; Ahabchane, N. E. H.; Es-Safi, N. E.; Garrigues, B.; Essassi, E. M. Spectroscopy Lett. 2007, 40, 741.

4. Sakata, G.; Makino, K.; Karasawa, Y. Heterocycles 1988, 27, 2481.

5. (a) Dell, A.; William, D. H.; Morris, H. R.; Smith, G. A.; Feeney, J.; Roberts, G. C. K. J. Am. Chem. Soc. 1975, 97, 2497. (b) Heravi, M. M.; Bakhtiari, K.; Tehrani, M. H.; Javadi, N. M.; Oskooie, H. A. ARKIVOC 2006, (xvi), 16. (c) Raw, S. A.; Wilfred, C. D.; Taylor, R. J. K. Chem. Commun. 2003, 18, 2286.

6. (a) Kumar, A.; Kumar, S.; Saxena, A.; De, A.; Mozumdar, S. Catal. Commun. 2008, 778. (b) Jaung, J. Y. Dyes and Pigments. 2006, 71, 45.

7. (a) Thomas, K. R. J.; Velusamy, M.; Lin, J. T.; Chuen, C. H.; Tao, Y. T. Chem. Mater. 2005, 17, 1860. (b) Dailey, S.; Feast, W. J.; Peace, R. J.; Sage, I. C.; Till, S.; Wood, E. 
L. J. Mater. Chem. 2001, 11, 2238. (c) Crossley, M. J.; Johnston, L. A. Chem. Commun. 2002, 1122. (d) Kazunobu, T.; Ryusuke, O.; Tomohiro, M. Chem. Commun. 2002, 212. (e) Sessler, J. L.; Maeda, H.; Mizuno, T.; Lynch, V. M.; Furuta, H. J. Am. Chem. Soc. 2002, 124, 13474. (f) Sascha, O.; Rudiger, F. Synlett 2004, 1509.

8. (a) Brown, D. J.; Quinoxalines: Supplement II, The Chemistry of Heterocyclic Compounds; Wiley: New Jersey, 2004. (b) Kim, S. Y.; Park, K. H.; Chung, Y. K. Chem. Commun. 2005, 1321.

9. Aparicio, D.; Attanasi, O. A.; Filippone, P.; Ignacio, R.; Lillini, S.; Mantellini, F.; Palacios, F.; Santos, J. M. J. Org. Chem. 2006, 71, 5897.

10. (a) Wu, Z.; Ede, N. J. Tetrahedron Lett. 2001, 42, 8115. (b) Singh, S. K.; Gupta, P.; Duggineni, S.; Kundu, B. Synlett 2003, 2147.

11. Antoniotti, S.; Donach, E. Tetrahedron Lett. 2002, 43, 3971.

12. Mao, L.; Sakurai, H.; Hirao, T. Synthesis 2004, 2535.

13. Zhao, Z.; Wisnoski, D. D.; Wolkenberg, S. E.; Leister, W. H.; Wang, Y.; Lindsley, C. W. Tetrahedron Lett. 2004, 45, 4873.

14. Bhosale, R. S.; Sarda, S. R.; Ardhapure, S. S.; Jadhav, W. N.; Bhusare, S. R.; Pawar, R. P. Tetrahedron Lett. 2005, 46, 7183.

15. More, S. V.; Sastry, M. N. V.; Wang, C. C.; Yao C. F. Tetrahedron Lett. 2005, 46, 6345 .

16. (a) Robinson, R. S.; Taylor, R. J. K. Synlett 2005, 1003. (b) Raw, S.A.; Wilfred, C. D.; Taylor, R. J. K. Org. Biomol. Chem. 2004, 2, 788. (c) Marques, C. S.; Moura, N.; Burke, A. J. Tetrahedron Lett. 2006, 47, 6049. (d) Cho, C. S.; Renb W. X.; Shimb, S. C. Tetrahedron Lett. 2007, 48, 4665. (e) Kotharkar, S. A.; Shinde, D. B. J. Iran. Chem. Soc. 2006, 3, 267. (f) Venkatesh, C.; Singh, B.; Mahata, P. K.; Junjappa, H. Org. Lett. 2005, 7, 2169. (g) Steven, A. R.; Cecilia, D. W.; Richard, J. K. T. Chem. Commun. 2003, 2286. (h) More, S. V.; Sastry, M. N. V.; Yao, C. F. Green Chem. 2006, 8, 91. (i) Heravi, M. M.; Taheri, S.; Bakhtiari, K.; Oskooie, H. A. Catal. Commun. 2007, 8, 211. (j) Huang, T.; Wang, R.; Shi, L.; Lu, X. Catal. Commun. 2008, 9, 1143. (k) Heravi, M. M.; Bakhtiari, K.; Bamoharram, F. F.; Tehrani, M. H. Monatsh Chem. 2007, 138, 465. (1) Darabi, H. R.; Mohandessi, S.; Aghapoor, K.; Mohsenzadeh, F. Catal. Commun. 2007, 8, 389. (m) Minsky, A.; Cohen, Y.; Rabinovitz, M. J. Am. Chem. Soc. 1985, 107, 1501 .

17. Feng, J; Liu, Y.; Meng, Q.; Liu, B. Synth. Commun. 1998, 28, 193.

18. Yavari, I.; Islami, M. R. J. Chem. Res. (S) 1998, 166.

19. Islami, M. R.; Abedini, J.; Fatemi, S. J.; Hassani, Z.; Amiry, A. Synlett 2004, 10, 1707.

20. Kalantari, M.; Islami, M. R.; Hassani, Z.; Saidi, K. ARKIVOC 2006, (x), 55.

21. Kappe, C. O. Angew. Chem. Int. Ed. 2004, 43, 6250.

22. Vogel, A. I.; Tatchell, A. R.; Furnis, B. S.; Hannaford, A. J.; Smith, P. W. G. Vogel's Textbook of Practical Organic Chemistry, 5th Edn. Wiley: New York, 1989; pp 10441045 . 